\title{
Investigating the Fabrication Imperfections of Plasmonic Nanohole Arrays and Its Effect on the Optical Transmission Spectra
}

\author{
Amr M. Mahros ${ }^{1,2}$ and Marwa M. Tharwat ${ }^{3}$ \\ ${ }^{1}$ Department of Physics, King Abdulaziz University, Jeddah 21432, Saudi Arabia \\ ${ }^{2}$ Department of Engineering Mathematics and Physics, Alexandria University, Alexandria 21544, Egypt \\ ${ }^{3}$ Department of Electrical Engineering, King Abdulaziz University, Jeddah 21432, Saudi Arabia
}

Correspondence should be addressed to Amr M. Mahros; amr.mahros@mena.vt.edu

Received 24 November 2014; Revised 26 February 2015; Accepted 27 February 2015

Academic Editor: Meng Lu

Copyright (C) 2015 A. M. Mahros and M. M. Tharwat. This is an open access article distributed under the Creative Commons Attribution License, which permits unrestricted use, distribution, and reproduction in any medium, provided the original work is properly cited.

\begin{abstract}
We investigate the extraordinary optical transmission spectra of thin gold films perforated with imperfect nanohole arrays using the finite difference time domain (FDTD) method. Exponential shapes for the nanohole sidewalls are used. To the best of our knowledge, such investigation of transmission spectra of imperfect nanohole arrays has not previously been demonstrated. It was found that the asymmetry between the two openings of the circular nanoholes or bending to their sidewalls strongly modifies both the intensity and resonance positions of the transmission spectra. Furthermore, the results of this study assist in explaining the technicality of extraordinary optical transmission phenomenon and why some experimental results on transmission differ from those expected.
\end{abstract}

\section{Introduction}

Metallic film perforated with periodic nanohole arrays is considered as one of the most interesting plasmonic structures and received a significant amount of attention. The extraordinary optical transmission spectra (EOT) of such structures have been the focus of considerable research activities. Discovery of EOT was first reported by Ebbesen in 1998 [1]. Numerous amounts of theoretical and experimental work about these structures are carried out by current researchers about investigating their optical properties.

The extraordinary behaviors, of the perforated metallic film, are largely due to the presence of surface plasmons (SPs), which can be excited by illuminating the film using a Gaussian beam. It was found in the literature that the coupling/decoupling phenomenon between SPs of patterned metallic film and incident light results in appearance of multiple resonance peaks in the transmission spectrum [2].

The EOT can be tuned through many physical and geometrical parameters including the hole shape, size, and depth as well as film material, substrate refractive index, and array period. Arrays with simple aperture shapes can be tuned to resonate at much shorter wavelengths than those of complex hole shapes, such as annular, cross-shaped, and $\mathrm{X}$-shaped apertures. However, more tunability had been achieved by applying a magnetic field [3-9].

The key parameters of theses plasmonic structures are metal type, holes shape and dimensions, refractive index of surrounding environment, and structural periodicity which have significant impact on the resonance wavelength and its intensity. However, the impact of fabricating imperfect nanoholes on the transmission spectrum has not yet been investigated in the literature. Nanoholes fabrication imperfections may result in disparity between their two apertures or bending to their sidewalls.

Surface plasmons (SPs) and EOT have been recently employed in a myriad of applications including biomedical sensing, efficient solar cells, fluorescence microscopy, photothermal therapy, and fabrication of nanoantennas [10-12]. 


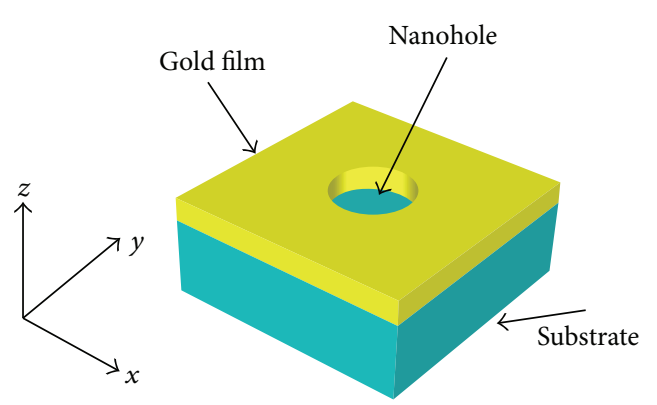

(a)

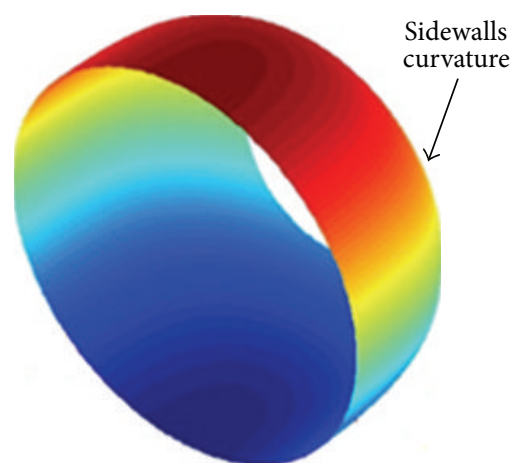

(b)

FIgURE 1: (a) A unit cell of a circular nanohole array perforated in a gold film. (b) Imperfect nanohole of asymmetric openings and sidewalls of exponential shape.

In this paper, we use the 3D finite difference time domain (FDTD) method to investigate the optical transmission spectrum of gold films patterned with imperfect circular nanoholes. We study the impact of changing the shape of imperfection on transmission spectra as a function of the nanohole depth, sidewalls tapering coefficient, and the imperfection ratio of asymmetric openings, showing that large modification can be achieved with realistic parameters. The impacts of independently varying these parameters are separately considered in our simulations, and the results are summarized in the following subsections.

This paper is organized as follows: the reported structure and FDTD simulation parameters are described in Section 2. Section 3 represents the optical transmission spectral responses of the designed plasmonic arrays and discussions for the effect of varying different key parameters. Finally, Section 4 provides conclusions of the obtained results.

\section{Structure Description and FDTD Simulation}

In this work, optical transmission spectra of the reported plasmonic imperfect nanohole arrays are obtained by solving Maxwell's equations of different materials using the FDTD algorithm. The FDTD method is applied by using the OptiFDTD simulation tool from Optiwave, Inc. The designed plasmonic structure layout is a circular nanohole array perforated in gold thin film "sandwiched" between glass substrate and air cladding as shown in Figure 1(a). The structural periodicity was $500 \mathrm{~nm}$ and the gold film thickness was $200 \mathrm{~nm}$.

In Cartesian coordinates $x, y$, and $z$ periodic boundary conditions were used in the $x$ and $y$ directions, while anisotropic perfect matching layer was used in the $z$-direction to serve as absorbing boundary condition. The frequency dependent relative permittivity $\varepsilon_{r}(\omega)$ of the dispersive gold film is characterized by Lorentz-Drude model:

$$
\varepsilon_{r}(\omega)=\varepsilon_{\infty}+\sum_{m=1}^{N} \frac{f_{m} \omega_{o m}^{2}}{\omega_{o m}^{2}-\omega^{2}+i \omega \Gamma_{m}},
$$

where $\varepsilon_{\infty}$ denotes the permittivity at infinite frequency, $f_{m}$ is a function of position specifying the oscillator strengths, and $\Gamma_{m}$ is the damping coefficient. The incident wave frequency and the resonant frequencies are, respectively, represented by $\omega$ and $\omega_{o m}$. For the substrate layer, $\varepsilon(\omega)$ was assumed as $n^{2}(n$ is the refractive index).

In order to realize a broadband simulation on the dispersive gold film, Gaussian modulated electromagnetic plane wave source was used. The continuous waves are centred at $680 \mathrm{~nm}$, linearly polarized in $y$-direction, and convoluted with a Gaussian envelope function. The light pulse in time domain has an offset time of $0.8 \times 10^{-14} \mathrm{~s}$ and half width of $1 \times$ $10^{-14} \mathrm{~s}$.

The simulation was performed at normal incidence $(z$ direction) of the plane wave through the nanohole arrays. The calculation mesh resolution was as high as $5 \mathrm{~nm}(<0.1 \lambda)$. The simulation runs for 5,000 time steps for a calculation time of $100 \mathrm{fs}$. An $x-y$ observation area will perform and calculate the transmission spectral analysis at $200 \mathrm{~nm}$ away from the gold/substrate interface.

Figure 1(b) shows the shape of the imperfect hole used during this simulation. Exponential shape for the nanohole sidewalls is used to express imperfection according to the following equation:

$$
R(Z)=R_{S}+\frac{R_{E}-R_{S}}{e^{-\alpha}-1}\left(e^{(-\alpha / d) Z}-1\right)
$$

where $R(z)$ represents the radius of the nanohole along its axis at different positions over the gold film of thickness $d$ such that $z=0$ and $z=d$ at the air/gold and the gold/substrate interfaces, respectively. The nanohole openings radii at the air/gold and the gold/substrate interfaces are, respectively, denoted by $R_{S}$ and $R_{E}$. The sidewalls exponential tapering coefficient is represented by $\alpha$. Figure 2 shows some examples for the shape of the nonlinear exponential tapered sidewalls at different values of $\alpha$ when $R_{S}=200 \mathrm{~nm}, d=200 \mathrm{~nm}$, and $R_{E}=125 \mathrm{~nm}$. The imperfection ratio is defined as ratio of the difference between the two openings' radii to the radius of the nanohole at the gold/substrate interface $\left(\delta R / R_{E}\right)$. 


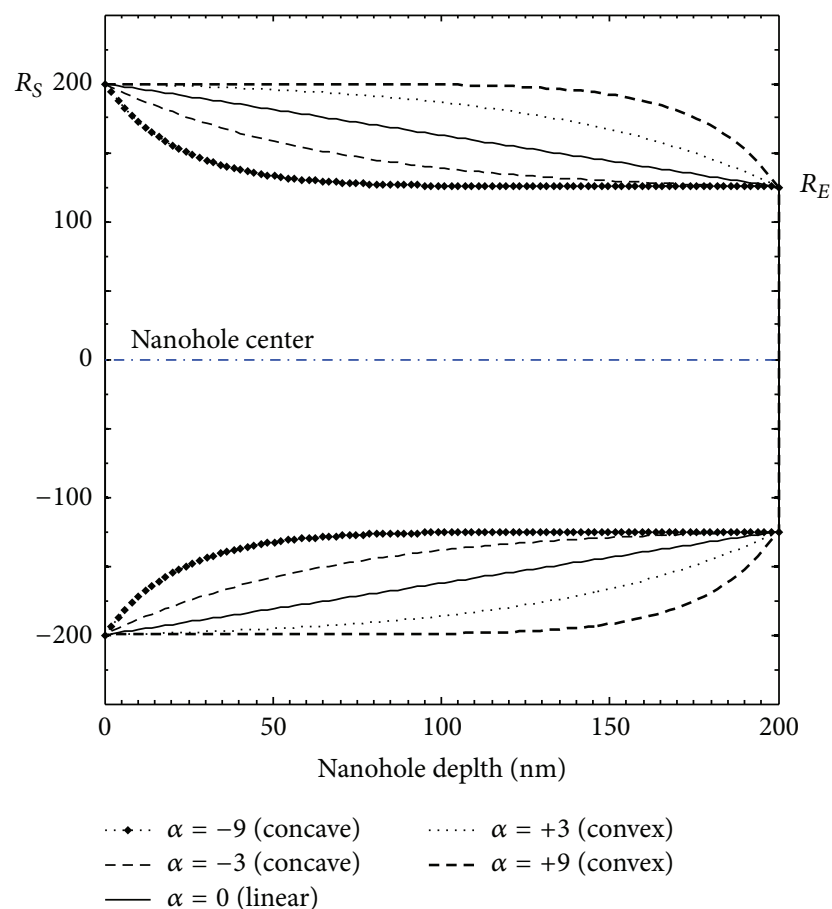

FIGURE 2: Examples for the shape of the exponential tapered sidewalls at different values of $\alpha$.

\section{Optical Transmission Spectral Responses of Nanohole Arrays}

In our simulations, using the FDTD method, we looked at the optical transmission spectrum of the reported plasmonic imperfect nanohole array as a function of different structural parameters such as the sidewalls exponential tapering coefficient, the imperfection ratio between the two asymmetric openings, and nanohole depth. The impacts of independently varying these parameters are separately considered in our simulations, and the results are summarized in the following subsections.

3.1. Tapering Coefficient. In this subsection, we investigate modifying the transmission spectra of the gold-perforated imperfect nanohole arrays by bended sidewalls due to fabrication imperfections. The sidewalls exponential tapering coefficient was changed from -9 to 9 passing through zero associated with linearly tapered sidewalls. During this study, the nanohole depth and opening radii at air/gold and the gold/substrate are kept constants at $200 \mathrm{~nm}, 125 \mathrm{~nm}$, and $200 \mathrm{~nm}$, respectively.

Figure 3 shows the transmission spectra of the designed structure at different values of the tapering coefficient $\alpha$. The results of Figure 3 exhibit the presence of two groups of resonance. The first group $R_{S}=200 \mathrm{~nm}$ is located at wavelengths less than $800 \mathrm{~nm}$, while the second group "P2" is found at wavelengths larger than $800 \mathrm{~nm}$.

It was found in the literature that these two sets of resonance wavelengths are associated with the air/gold and the gold/substrate interfaces. At normal incidence, the SP
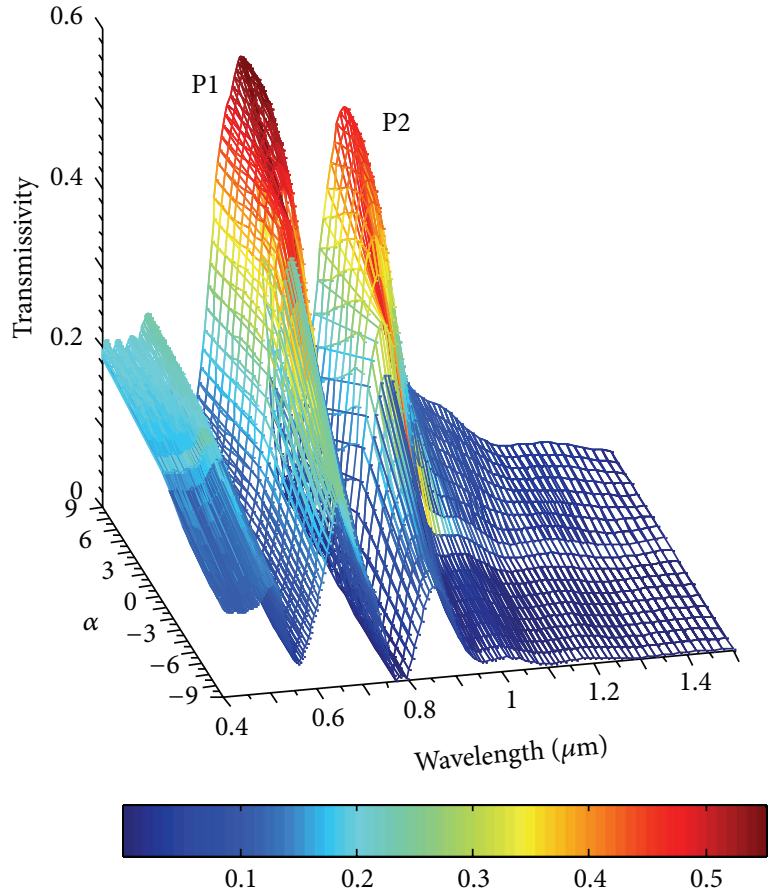

FIGURE 3: Optical transmission spectra of gold thin film perforated with imperfect nanoholes at different values of $\alpha$.

resonance wavelengths $\lambda_{\mathrm{SP}}$ of a circular nanohole array can be approximated by the following equation:

$$
\lambda_{\mathrm{SP}}(i, j)=\operatorname{Re}\left(\frac{\Lambda}{\sqrt{i^{2}+j^{2}}} \sqrt{\frac{\varepsilon_{d} \varepsilon_{m}}{\varepsilon_{d}+\varepsilon_{m}}}\right),
$$

where $\varepsilon_{d}$ and $\varepsilon_{m}$, respectively, represent the relative permittivities of the adjacent medium and the metal and $(i, j)$ are integers. $\Lambda$ represents the structural periodicity value. The $\mathrm{SP}$ resonance wavelength in the range of $650-700 \mathrm{~nm}$ "P1" can be assigned to $\lambda_{\mathrm{SP}}(1,1)$ of the air/gold interface, while the resonance wavelength found at values larger than $800 \mathrm{~nm}$ "P2" is related to $\lambda_{\mathrm{SP}}(1,0)$ the gold/substrate interface.

Figure 4 shows the impact of changing the tapering coefficient on the position of the resonance wavelengths and the corresponding transmission peaks associated to "P1" and "P2" resonances.

As demonstrated in Figures 4(a) and 4(b), as $\alpha$ increases, the resonance wavelengths exhibit a clear redshift. The red shift may be attributed to the change of the effective depth of the nanohole. As $\alpha$ increases, the effective length of the resonator increases as shown in Figure 2. For higher absolute values of the exponential tapering coefficient, the sidewalls show monotony surface resulting in a fixed effective length of the resonator and constant resonance wavelengths.

Additionally, Figures 4(c) and 4(d) show the impact of changing $\alpha$ on the transmission peak intensity of "P1" and "P2", respectively. The illustrated results demonstrate that the peak intensity values of "P1" and "P2" monotonically increase 


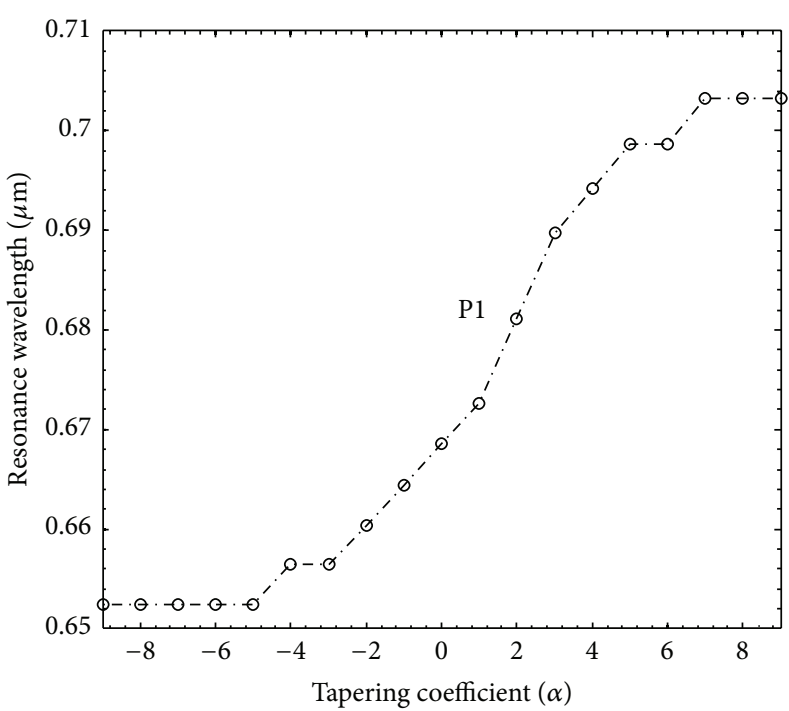

(a)

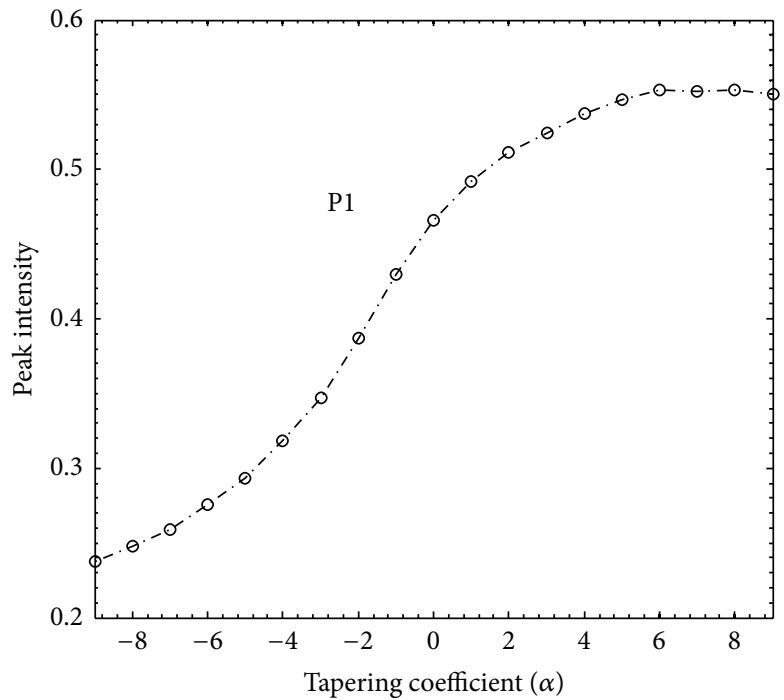

(c)

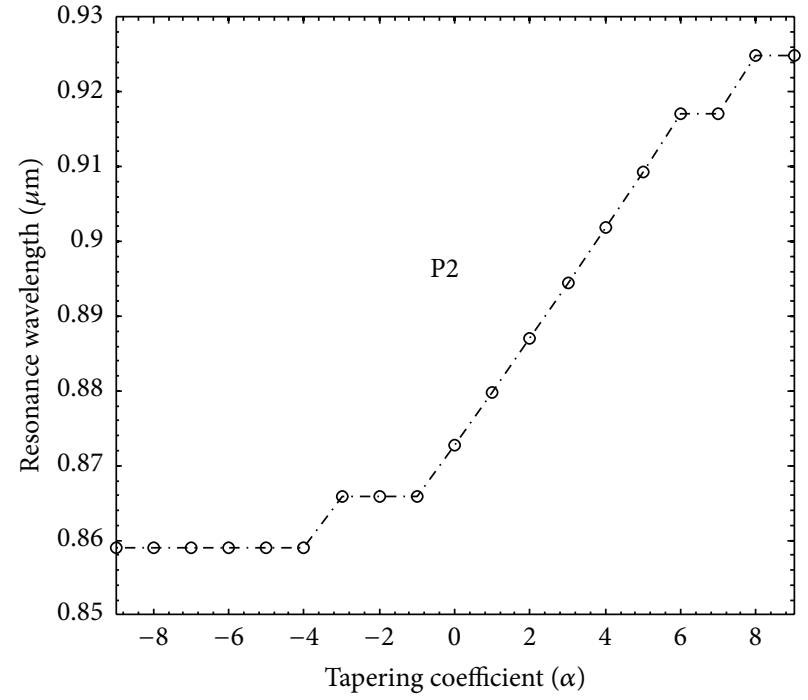

(b)

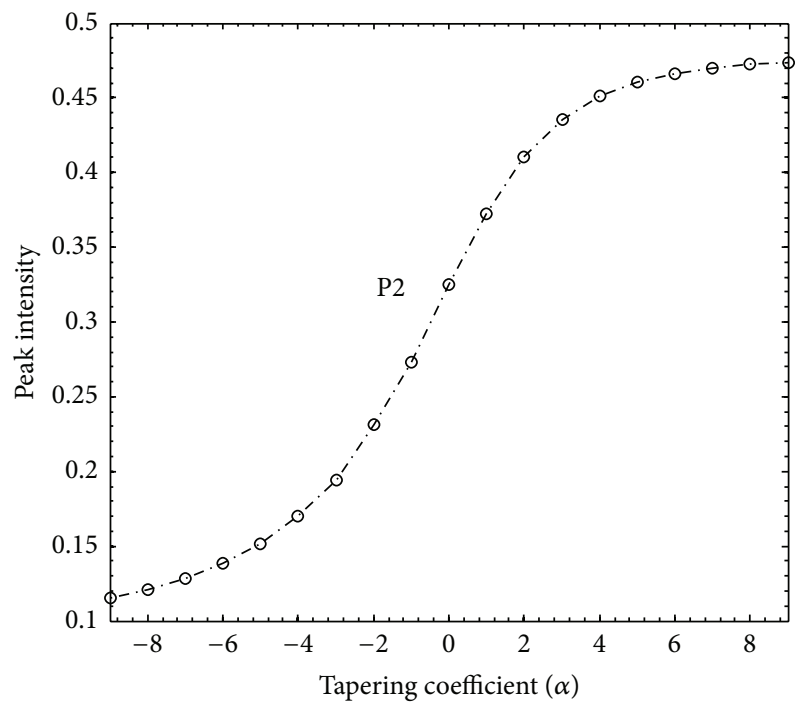

(d)

FIGURE 4: Effect of changing $\alpha$ on the position of resonance wavelengths of (a) "P1" and (b) "P2." Impact of changing $\alpha$ on the transmission peak intensity of (c) "P1" and (d) "P2."

with increasing $\alpha$ till it saturates at higher values of the tapering coefficient.

This happens because positive values of $\alpha$ provide convex metallic sidewalls which collect more optical energy to funnel to the other side of the metal surface. On the other hand, negative values of $\alpha$ define concave metallic sidewalls which are associated with higher reflection and lower transmission.

In comparison to the perfect circular nanohole arrays, Bethe predicted that the transmission peak intensity values of such structures are proportional to $R^{4}$. Therefore, the difference in peak transmission coefficient can perhaps be attributed to the differences in "effective" hole radius.

3.2. Imperfection Ratio. In this subsection, we investigate the effect of changing the imperfection ratio $\left(\delta R / R_{E}\right)$, up to $80 \%$, on the transmission spectra of the gold-perforated imperfect nanohole arrays. During this investigation, the nanohole depth and radius at gold/substrate interface remain constants at $200 \mathrm{~nm}$ and $125 \mathrm{~nm}$, respectively. Note that changing the imperfection ratio, from 0 to 0.8 , results in a change of enter opening radius from $125 \mathrm{~nm}$ to $225 \mathrm{~nm}$. However, the exit opening radius is fixed at $125 \mathrm{~nm}$.

Figure 5 demonstrates the modifying transmission spectra through gold-perforated imperfect nanohole array due to the change of $\delta R / R_{E}$. These simulations were performed for two arrays with different sidewalls tapering coefficients such that Figures 5(a) and 5(b) are illustrated for $\alpha=4$ and $\alpha=8$, respectively. The radius of the nanohole along its axis decreases between the air/gold interface and the gold/substrate interface following the exponential profile. 


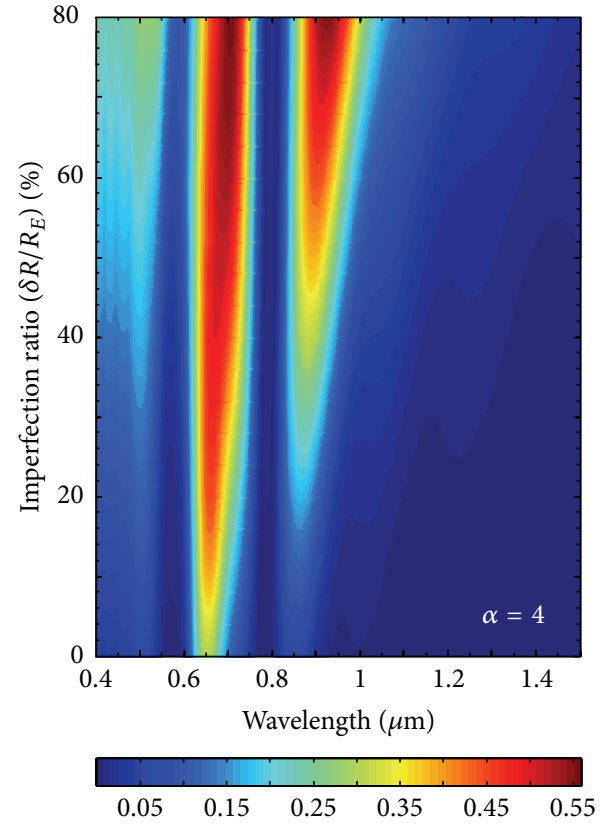

(a)

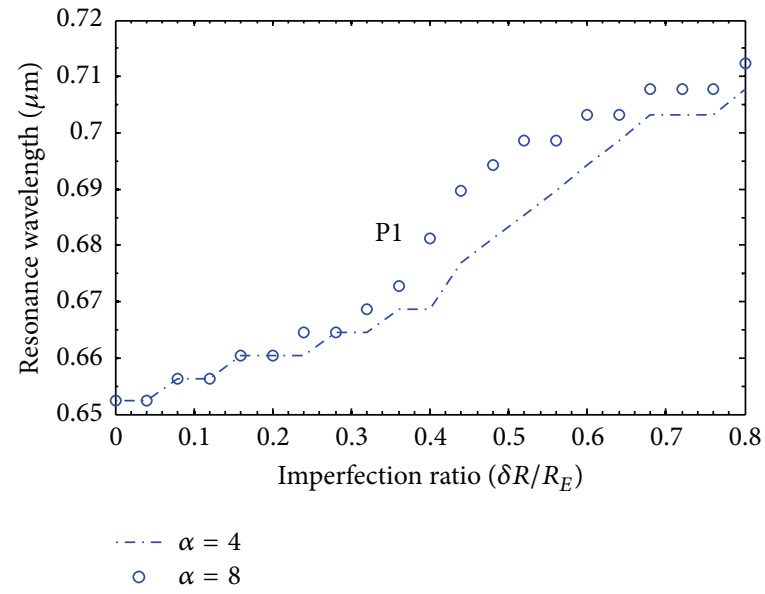

(c)

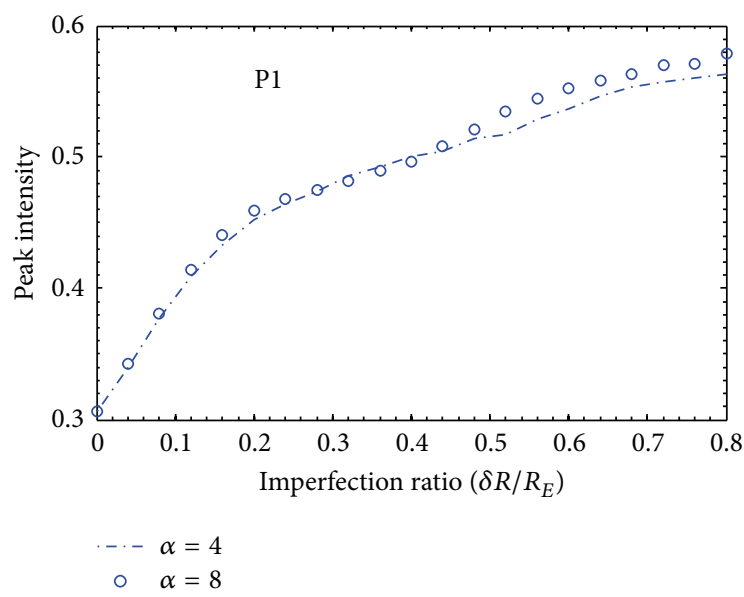

(e)

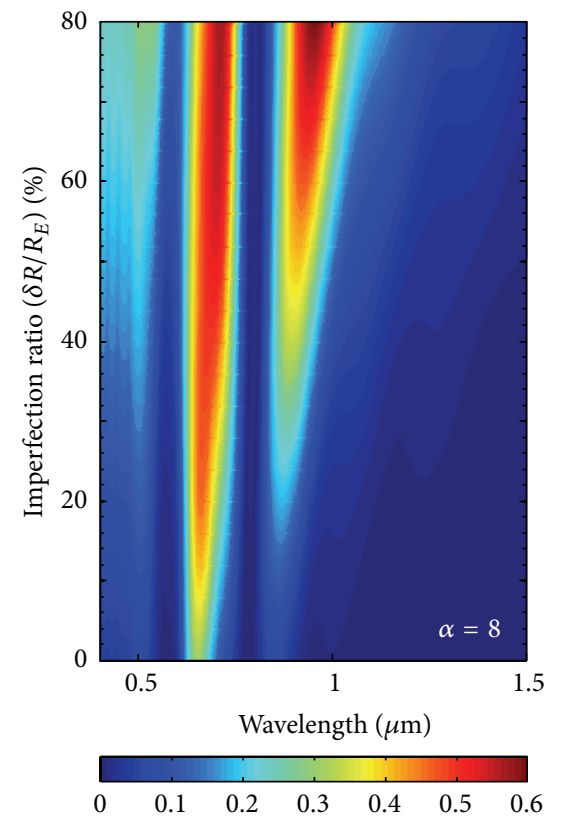

(b)

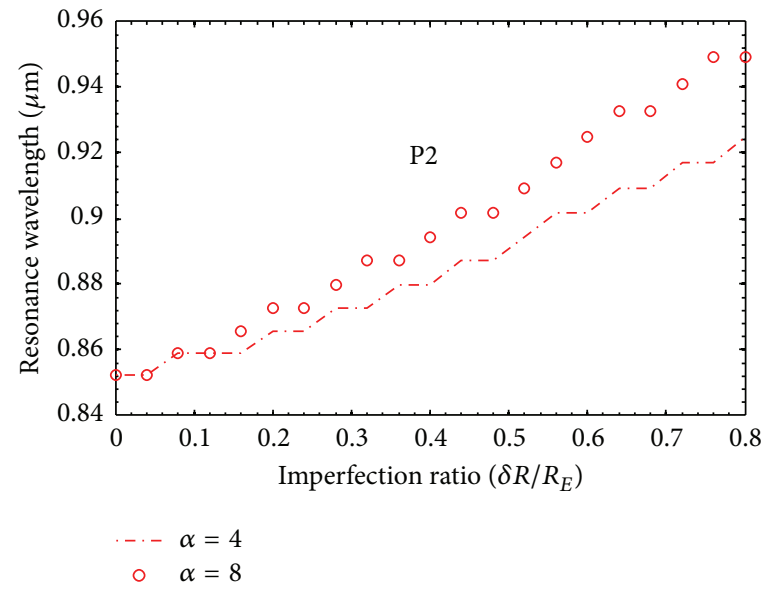

(d)

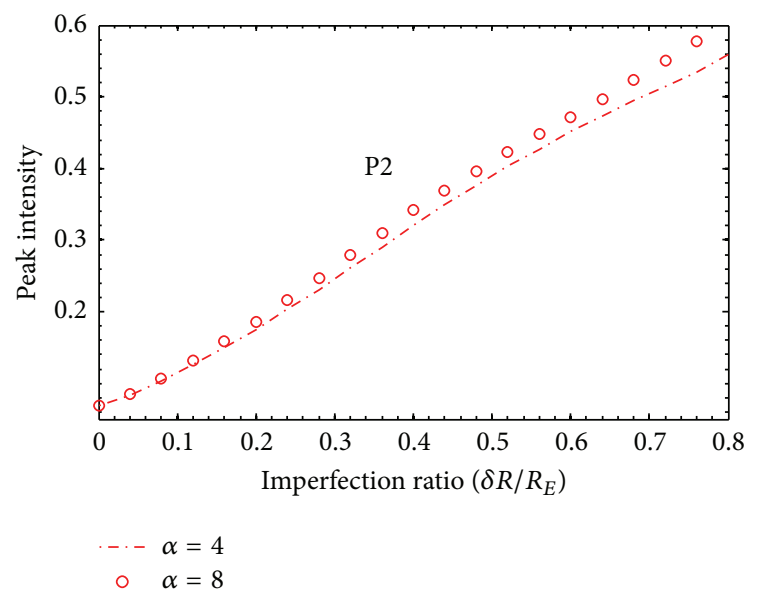

(f)

FIGURE 5: Effect of changing the imperfection ratio on (a) the transmission spectra $(\alpha=4)$, (b) the transmission spectra $(\alpha=8)$, the position of resonance wavelengths of (c) "P1" and (d) "P2," and the transmission peak intensity of (e) "P1" and (f) "P2." 


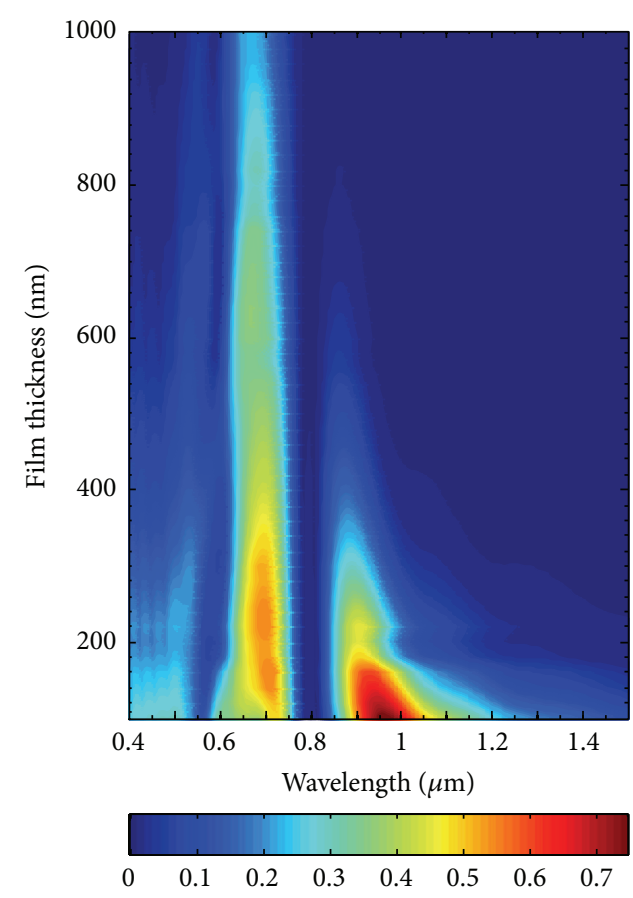

(a)

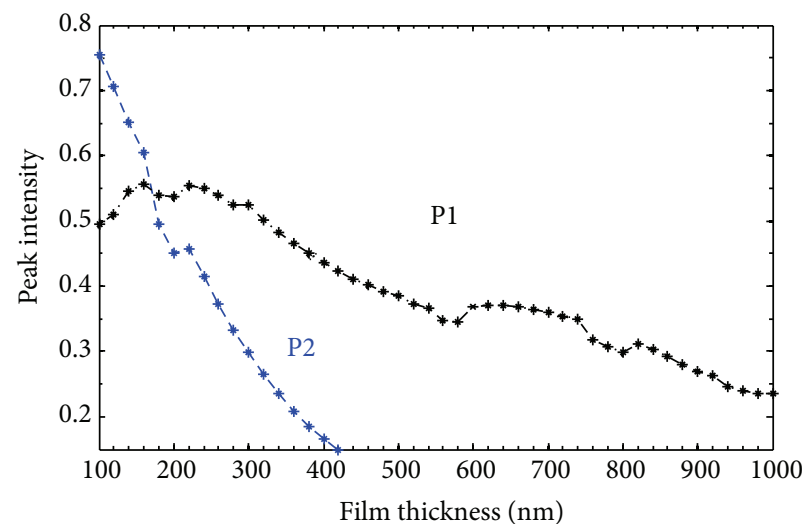

(b)

FIGURE 6: Effect of changing the imperfect nanohole depth on (a) the transmission spectra and (b) the transmission peak intensity of "P1" and "P2."

Imperfect nanohole array with $\alpha=4$ shows a higher decreasing rate than that of one with $\alpha=8$.

It can be seen from Figures 5(c) and 5(e) that the peak transmittance and resonance wavelength of "P1" slightly change with increasing imperfection ratio. In sharp contrast, a clear red shift in the resonance position and dramatically ascent in the intensity of "P2" is achieved as shown in Figures 5(d) and 5(f).

As the imperfection ratio increases, the nonlinearity of the tapered exponential sidewalls increases. It is expected that increasing the nonlinearity magnifies the tunneling of light via coupled plasmonic modes localized in the nanohole. As the distance between the gold surfaces narrows, the localized plasmon polariton gets stronger. This might allow most of energy to flow inside the tapered nanohole and it is coupled with SP waves on the inner sidewalls. Increasing the confinement will results in red shift.

The impact of changing $\delta R / R_{E}$ on the resonance wavelength and the peak transmission of "P2" are almost double the effect on "P1." This may be attributed to the fact that the exponential profile shows higher slope and hence stronger impact on "P2" at the gold/substrate interface than that on "P1" at the air/gold interface.

Furthermore, as shown in Figure 2, the impact of the sidewalls tapering coefficient on the slope of the sidewalls appears at higher values of $z$ near the exit opening at gold/substrate interface. As a result, both the peak intensity and resonance wavelength of "P2" are notably influenced changing $\alpha$ as demonstrated in Figures 5(d) and 5(f). However, changing $\alpha$ almost has no effect on both the peak intensity and resonance wavelength of "P1" as illustrated in Figures 5(c) and 5(e).

3.3. Nanohole Depth. In this subsection, the behavior of optical transmission spectra of the reported plasmonic structure is presented with changing the distance between the two asymmetry openings. We use the following parameters: $\Lambda=$ $500 \mathrm{~nm}, R_{S}=200 \mathrm{~nm}, R_{E}=125 \mathrm{~nm}, \alpha=4$, and $d$ in the range from $100 \mathrm{~nm}$ to $1000 \mathrm{~nm}$. The impact of changing film thickness on the intensity and resonance positions of the transmission spectra is shown in Figure 6.

Figure 6(a) shows the two groups of peaks "P1" and "P2." For film thickness higher than $400 \mathrm{~nm}$, "P2" is totally suppressed and only "P1" is delivered. That will be useful in design of visible and near infrared photonic-based optical devices. Changing the film thickness almost has no impact on the resonance wavelengths of both "P1" and "P2." Figure 6(b) demonstrates that the transmission peak intensity of "P1" slightly decreases with increasing nanohole depth. In sharp contrast, the intensity of "P2" suffers sever degradation with increasing the depth.

For further investigation, the spatial distribution of the total magnetic and electric field intensities at the middle of the nanohole is demonstrated in Figure 7. In Figure 7(a), magnetic field distribution in $y z$ plane indicates that strong field is accumulating on the upper surface of the perfect nanohole. In sharp contrast, Figure 7(b) shows energy penetration into the imperfect nanohole with low reflections. The electric field distribution is localized on the air/gold interface of the perfect 


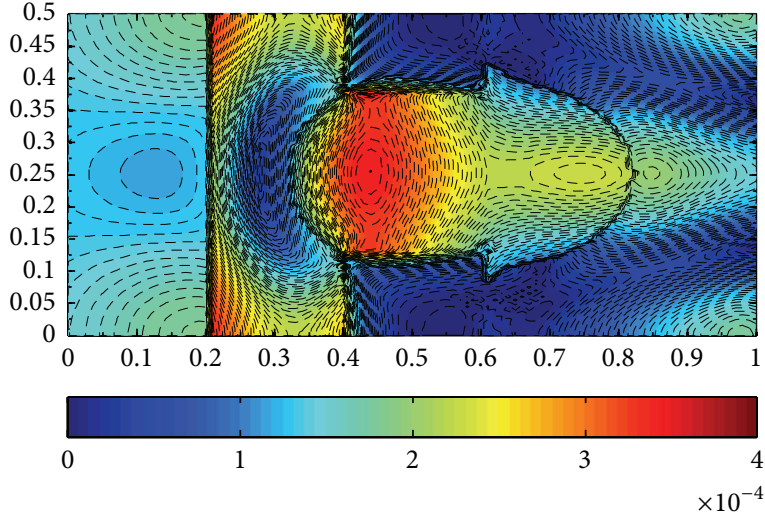

(a)

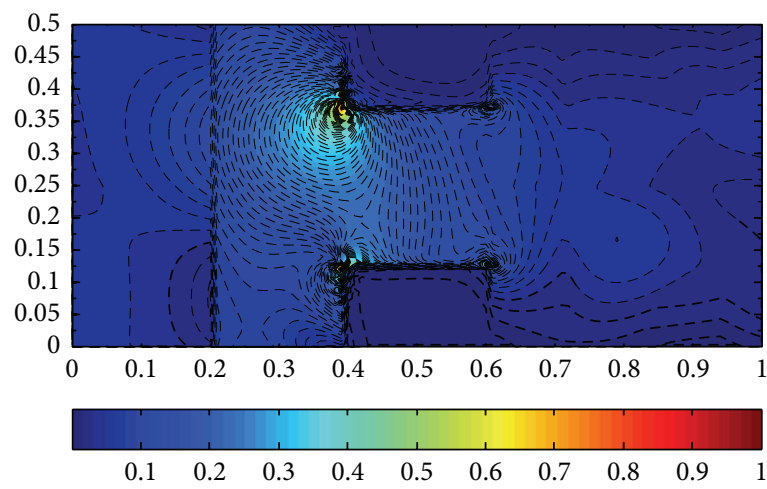

(c)

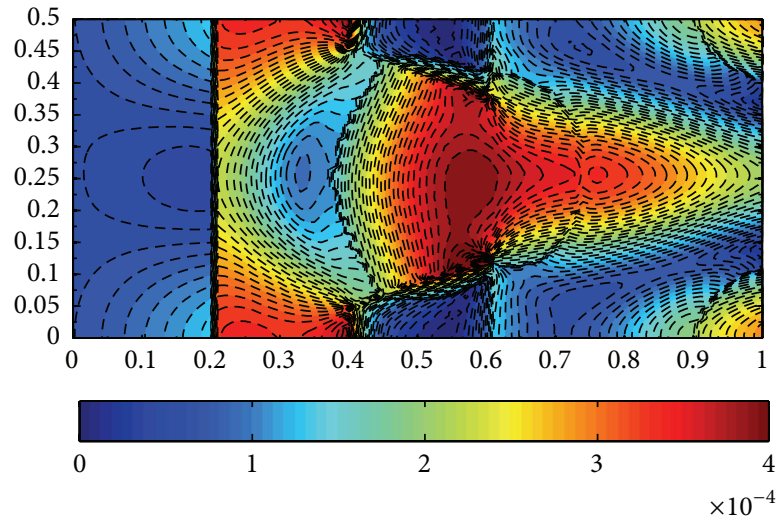

(b)

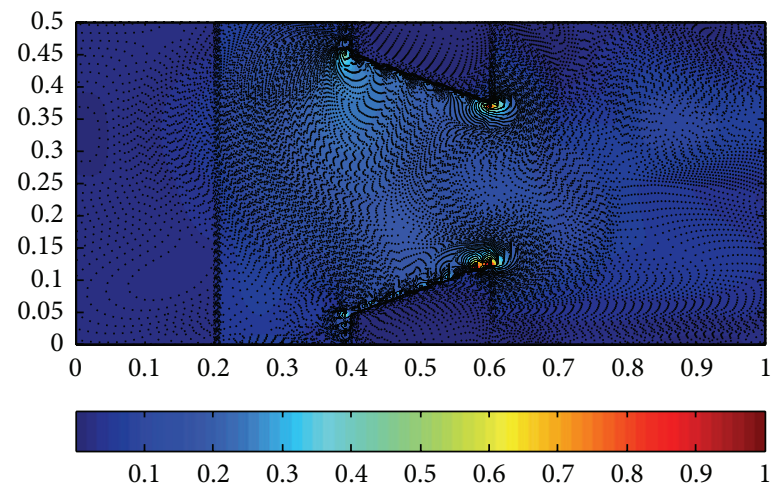

(d)

Figure 7: Spatial distribution of the total magnetic and electric field intensities in $y z$ plane at the middle of the nanohole.

nanohole as shown in Figure 7(c). However, large entering radius at the air/gold interface and tapered sidewalls of the imperfect nanohole lead to concentration of the electric field at narrow exiting radius at the gold/glass substrate.

\section{Conclusion}

In this work, we comprehensively investigate the EOT properties of the imperfect circular plasmonic nanohole arrays. Using the FDTD method, we investigate the impact of several key parameters including nanohole depth, ratio between the asymmetric openings, and sidewalls tapering coefficient on the transmission spectra of thin gold films perforated with imperfect nanohole arrays. A notable red shift in the resonance wavelength of the plasmonic wave at the gold/substrate interface is achieved with increasing either the sidewalls tapering coefficient or the imperfection ratio. However, increasing film thickness causes a notable degradation in the transmission intensity above $400 \mathrm{~nm}$.

\section{Conflict of Interests}

The authors declare that there is no conflict of interests regarding the publication of this paper.

\section{References}

[1] T. W. Ebbesen, H. J. Lezec, H. F. Ghaemi, T. Thio, and P. A. Wolff, "Extraordinary optical transmission through sub-wavelenght hole arrays," Nature, vol. 391, no. 6668, pp. 667-669, 1998.

[2] H. F. Ghaemi, T. Thio, D. E. Grupp, T. W. Ebbesen, and H. J. Lezec, "Surface plasmons enhance optical transmission through subwavelength holes," Physical Review B, vol. 58, no. 11, pp. 6779-6782, 1998.

[3] S. M. Orbons, M. I. Haftel, C. Schlockermann et al., "Dual resonance mechanisms facilitating enhanced optical transmission in coaxial waveguide arrays," Optics Letters, vol. 33, no. 8, pp. 821-823, 2008.

[4] L. Lin and A. Roberts, "Light transmission through nanostructured metallic films: coupling between surface waves and localized resonances," Optics Express, vol. 19, no. 3, pp. 26262633, 2011.

[5] R. Biswas, S. Neginhal, C. G. Ding, I. Puscasu, and E. Johnson, "Mechanisms underlying extraordinary transmission enhancement in subwavelength hole arrays," Journal of the Optical Society of America B: Optical Physics, vol. 24, no. 10, pp. 25892596, 2007.

[6] T. Vallius, J. Turunen, M. Mansuripur, and S. Honkanen, "Transmission through single subwavelength apertures in thin metal films and effects of surface plasmons," Journal of the Optical Society of America A: Optics and Image Science, and Vision, vol. 21, no. 3, pp. 456-463, 2004. 
[7] A. K. Azad, Y. Zhao, W. Zhang, and M. He, "Effect of dielectric properties of metals on terahertz transmission subwavelength hole arrays," Optics Letters, vol. 31, no. 17, pp. 2637-2639, 2006.

[8] M. J. Kofke, D. H. Waldeck, Z. Fakhraai, S. Ip, and G. C. Walker, "The effect of periodicity on the extraordinary optical transmission of annular aperture arrays," Applied Physics Letters, vol. 94, no. 2, Article ID 023104, 2009.

[9] A. Battula, S. Chen, Y. Lu, R. J. Knize, and K. Reinhardt, "Tuning the extraordinary optical transmission through subwavelength hole array by applying a magnetic field," Optics Letters, vol. 32, no. 18, pp. 2692-2694, 2007.

[10] R. Wannemacher, "Plasmon-supported transmission of light through nanometric holes in metallic thin films," Optics Communications, vol. 195, no. 1-4, pp. 107-118, 2001.

[11] G. C. des Francs, D. Molenda, U. C. Fischer, and A. Naber, "Enhanced light confinement in a triangular aperture: experimental evidence and numerical calculations," Physical Review B: Condensed Matter and Materials Physics, vol. 72, no. 16, Article ID 165111, 2005.

[12] I. Ashry, B. Zhang, S. V. Stoianov et al., "Probing the photonic density of states using layer-by-layer self-assembly," Optics Letters, vol. 37, no. 11, pp. 1835-1837, 2012. 

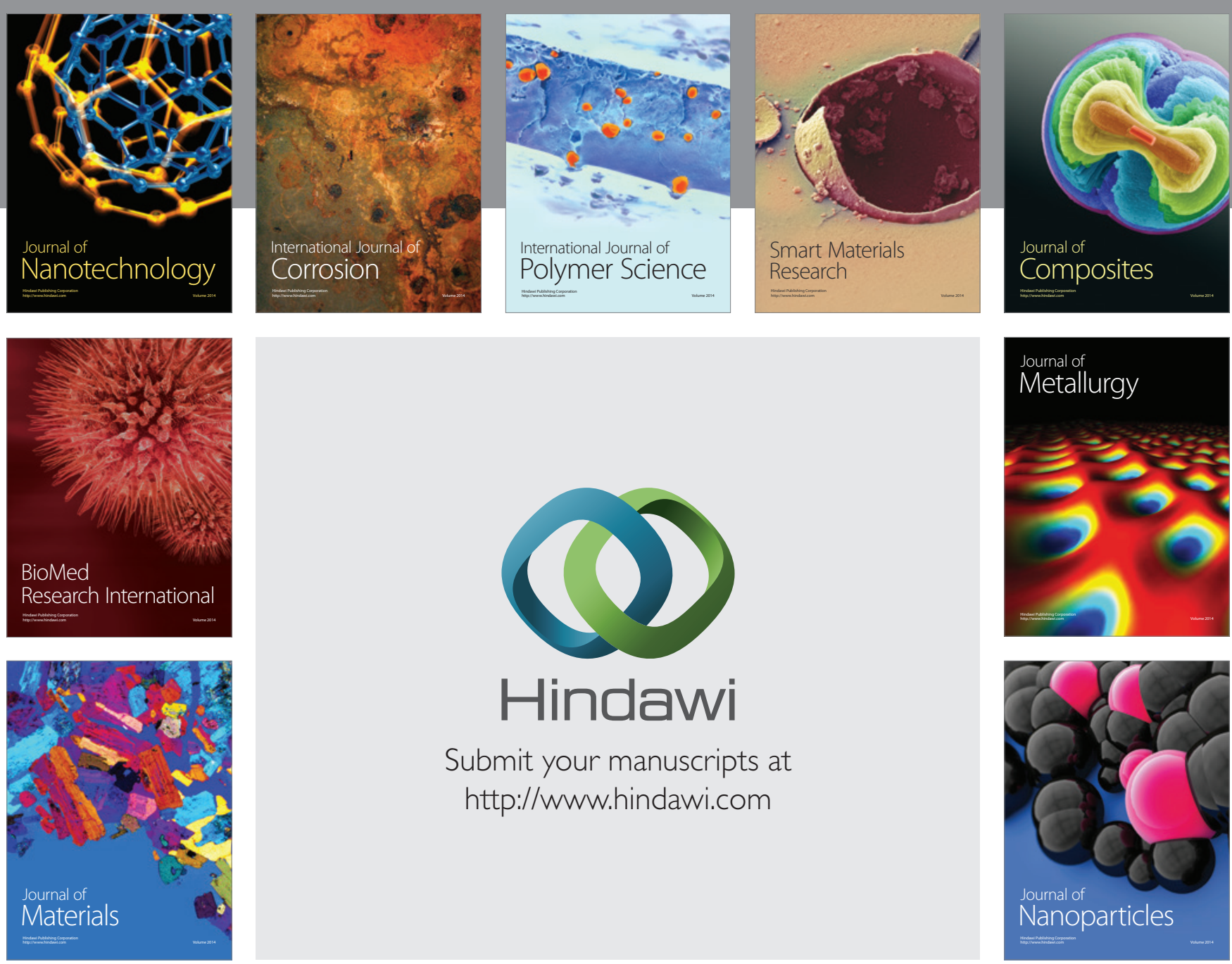

Submit your manuscripts at http://www.hindawi.com
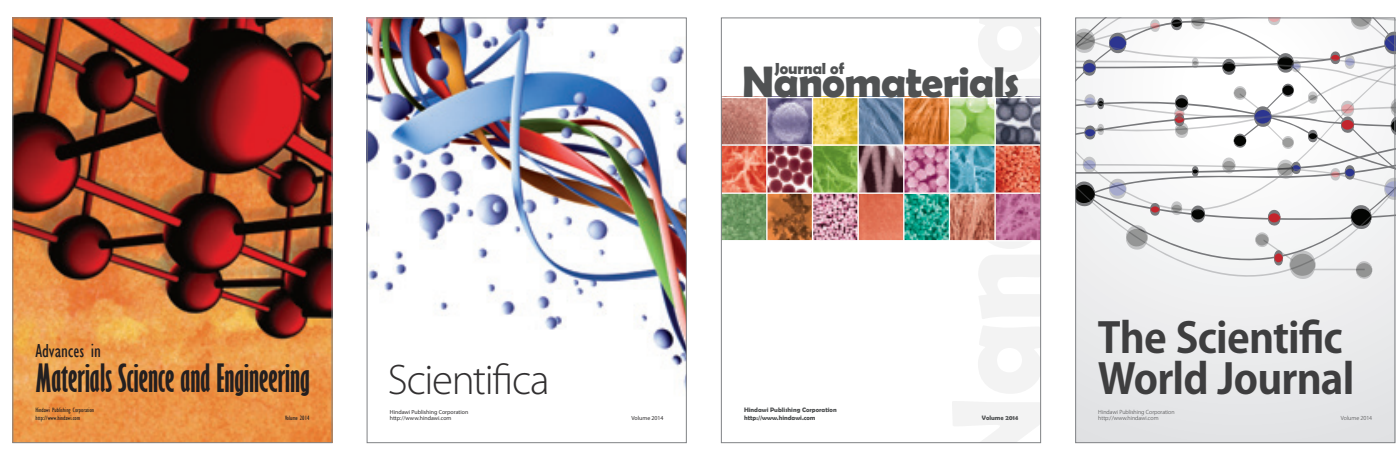

\section{The Scientific World Journal}
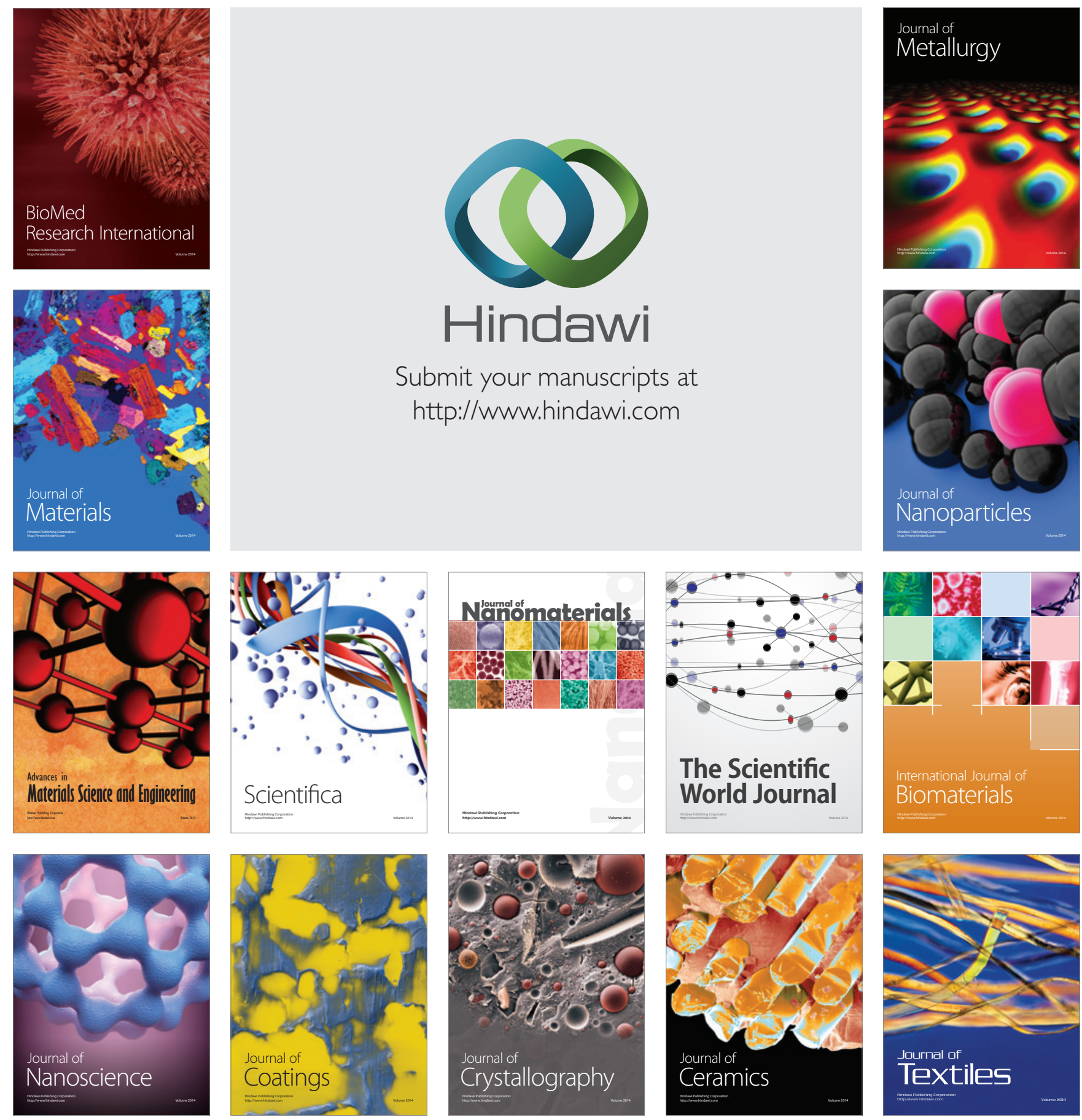